\title{
RESOLUTION IN HÖLDER SPACES OF AN ELLIPTIC PROBLEM IN AN UNBOUNDED DOMAIN
}

\author{
TARIK BERROUG, RABAH LABBAS and BOUBAKER-KHALED SADALLAH ${ }^{\circ}$
}

(Received 23 July 2004; revised 4 July 2005)

Communicated by A. J. Pryde

\begin{abstract}
In this paper we give new results concerning the maximal regularity of the strict solution of an abstract second-order differential equation, with non-homogeneous boundary conditions of Dirichlet type, and set in an unbounded interval. The right-hand term of the equation is a Hölder continuous function.
\end{abstract}

2000 Mathematics subject classification: primary 12H20, 34G10; secondary 35J25, 44A45.

Keywords and phrases: Hölder Spaces, abstract elliptic equation.

\section{Introduction}

This work is devoted to the study of the second-order abstract differential equation

$$
u^{\prime \prime}(t)+A u(t)=f(t), \quad t \in(0,+\infty),
$$

under non-homogeneous boundary conditions of Dirichlet type given by

$$
u(0)=\varphi, \quad u(+\infty)=0,
$$

where $\varphi$ and $f(t)$ belong to a complex Banach space $E$ and $A$ is a closed linear operator with domain $D(A)$ not necessarily dense in $E$.

For $l \in \mathbb{N}$, we denote by $B U C([0,+\infty[; E)$ the space of vector-valued functions with uniformly continuous and bounded derivatives up to order $l$ in $[0,+\infty[$ and by $C^{\sigma}([0,+\infty[; E)$ for $\sigma \in] 0,1$, the space of bounded and $\sigma$-Hölder continuous functions $f:\left[0,+\infty\left[\rightarrow E\right.\right.$, such that $\sup _{t \in[0,+\infty}\|f(t)\|_{E}<\infty$ and there exists $C>0$ such that for all $t, \tau \in[0,+\infty[$,

$$
\|f(t)-f(\tau)\|_{E} \leqslant C|t-\tau|^{\sigma},
$$

(C) 2006 Australian Mathematical Society 1446-7887/06 \$A2.00+0.00 
endowed with the norm

$$
\begin{aligned}
\|f\|_{C^{\sigma}([0,+\infty[; E)} & =\sup _{t \in[0,+\infty[}\|f(t)\|_{E}+\sup _{t \neq \tau} \frac{\|f(t)-f(\tau)\|_{E}}{|t-\tau|^{\sigma}} \\
& =\|f\|_{\infty}+[f]_{C^{\sigma}([0,+\infty[; E) .}
\end{aligned}
$$

For simplicity, we shall write $C^{\sigma}(E)$ instead of $C^{\sigma}([0,+\infty[; E)$.

In the present study, we are interested in the existence, the uniqueness and the maximal regularity of the strict solution $u$ in the Banach space $X=B U C([0,+\infty[; E)$, when the right-hand term $f$ is regular (Hölder continuous function).

We recall that $u \in B U C([0,+\infty[; E)$ is a strict solution of Problem (1.1)-(1.2) if

$$
u \in B U C([0,+\infty[; E) \cap B U C([0,+\infty[; D(A)),
$$

and $u$ satisfies (1.1) and (1.2).

Throughout this paper we assume that the resolvent of $A$ verifies the hypothesis that there exists $K>0$ such that for all $\lambda \geqslant 0$

$$
\left\|(A-\lambda I)^{-1}\right\|_{L(E)} \leqslant \frac{K}{1+\lambda} .
$$

Equations (1.1)-(1.2) can be illustrated by the following example of a Laplacian problem

$$
\begin{cases}\frac{\partial^{2} u}{\partial t^{2}}(t, x)+\frac{\partial^{2} u}{\partial x^{2}}(t, x)=f(t, x), & (t, x) \in(0,+\infty) \times(0,1), \\ u(0, x)=\varphi(x), \quad u(+\infty, x)=0, & x \in(0,1), \\ u(t, 0)=u(t, 1)=0 . & \end{cases}
$$

Indeed, in $E=C([0,1])$, we can choose the operator $A$ as follows:

$$
\begin{aligned}
D(A) & =\left\{v \in C^{2}([0,1]): v(0)=v(1)=0\right\} \subset E, \\
(A v)(x) & =v^{\prime \prime}(x), \quad v \in D(A) .
\end{aligned}
$$

Several authors have studied equation (1.1) under various (homogeneous or nonhomogeneous) boundary conditions, as well as in the case of variable operators, but on a finite interval. See, for instance, Krein [5], Sobolevskii [11], Véron [13], Kuyazyuk [6], Da Prato-Grisvard [9], Labbas [7].

Our approach is based on the direct use of the operational calculus and of Dunford's integrals as in Da Prato-Grisvard [9]. We make use of the real Banach interpolation spaces $D_{A}(\theta,+\infty)$, between $D(A)$ and $E$, which are well-known in many concrete cases and can be characterized by

$$
D_{A}(\theta,+\infty)=\left\{\xi \in E: \sup _{t>0}\left\|t^{\theta} A(A-t I)^{-1} \xi\right\|_{E}<\infty\right\}
$$


where $\theta \in(0,1)$ (see Grisvard [4]).

Assumption (1.3) does not imply that $A$ is an infinitesimal generator of an analytical semigroup. However it allows us to define $(-A)^{1 / 2}$, (for details, see Balakrishnan [1]). We do not use this fractional power of the operator nor the techniques of semigroups estimates generated by them as in Krein [5].

Our main results are the following.

THEOREM 1.1. Let $\varphi \in D(A)$ and $f \in C^{2 \theta}(E)$, with $\theta \in(0,1 / 2)$ such that

$$
f(0)-A \varphi \in \overline{D(A)} \text {. }
$$

Then Problem (1.1)-(1.2) admits a unique strict solution.

THEOREM 1.2. Let $\varphi \in D(A)$ and $f \in C^{2 \theta}(E)$, with $\theta \in(0,1 / 2)$ such that

$$
f(0)-A \varphi \in D_{A}(\theta,+\infty) \text {. }
$$

Then the unique strict solution of (1.1)-(1.2) satisfies the property of maximal regularity $A u(\cdot), u^{\prime \prime}(\cdot) \in C^{2 \theta}(E)$.

If $f$ is in a $L^{p}$-Lebesgue space, we can prove that the same representation given in (2.1) leads to the existence of a strict solution, because Lebesgue spaces satisfy the so-called UMD property. This no longer holds true for Hölder spaces; so the function needs more regularity (see, for instance, Favini et al. [3]).

The paper is organized as follows. In Section 2, we give the natural representation of the solution $u$ to Problem (1.1)-(1.2) by using the operational calculus and Dunford's integral. In Section 3, we give necessary and sufficient conditions on $\varphi$ and $A \varphi-f(0)$ to obtain the maximal smoothness of the solution $u$ given by Dunford's integral when $f$ is Hölderian. In Section 4, we present an example, to which our abstract results can be applied.

\section{Construction of the solution}

If $A$ is a complex scalar $z$ such that $\operatorname{Re} \sqrt{-z}$ is positive, then the solution of (1.1)-(1.2) is given by

$$
u(t)=e^{-\sqrt{-z} t} \varphi-\int_{0}^{+\infty} k(t, s) f(s) d s,
$$

where

$$
k(t, s)= \begin{cases}\frac{e^{-\sqrt{-z} t} \sinh \sqrt{-z} s}{\sqrt{-z}} & 0 \leqslant s \leqslant t, \\ \frac{e^{-\sqrt{-z} s} \sinh \sqrt{-z} t}{\sqrt{-z}} & s \geqslant t .\end{cases}
$$


Here $\sqrt{-z}$ is the analytic determination defined by $\operatorname{Re} \sqrt{-z}>0$. In the general case, it is well known that Hypothesis (1.3) implies the existence of $\delta_{0} \in(0, \pi / 2)$ and $\epsilon_{0}>0$ such that the resolvent set of $A$ contains a sectorial domain of the form

$$
S_{\epsilon_{0}, \delta_{0}}=S=\left\{\lambda \in \mathbb{C}^{*}:|\arg \lambda| \leqslant \delta_{0}\right\} \cup \overline{B\left(O, \epsilon_{0}\right)},
$$

where $B\left(O, \epsilon_{0}\right)$ is an open ball of radius $\epsilon_{0}$. If $\gamma$ denotes the sectorial boundary curve of $S_{\epsilon_{0}, \delta_{0}}$ oriented positively, remaining in $\rho(A) \backslash \mathbb{R}_{+}$, and defined by

$$
\gamma=\left\{z \in \mathbb{C}:|z| \geqslant \epsilon_{0} \text { and }|\arg z|=\delta_{0}\right\} \cup\left\{z=\epsilon_{0} e^{i v}: \delta_{0} \leqslant v \leqslant 2 \pi-\delta_{0}\right\},
$$

then the natural representation of the solution of (1.1)-(1.2), in the abstract case, is given by Dunford's integral

$$
\begin{aligned}
u(t)= & \frac{1}{2 i \pi} \int_{\gamma} e^{-\sqrt{-z} t}(A-z I)^{-1} \varphi d z \\
& -\frac{1}{2 i \pi} \int_{\gamma} \int_{0}^{+\infty} k(t, s)(A-z I)^{-1} f(s) d s d z .
\end{aligned}
$$

These integrals converge absolutely for every $t \in(0,+\infty)$. Indeed, we have for $z \in \gamma,\left|e^{-\sqrt{-z} t}\right| \leqslant e^{-\operatorname{Re} \sqrt{-z} t}=e^{-c_{0}|z|^{1 / 2} t}$, where $c_{0}=\cos \left(\left(\pi-\delta_{0}\right) / 2\right)$. On the other hand, for any $f \in X$, we see that

$$
\left\|\int_{0}^{+\infty} k(t, s)(A-z I)^{-1} f(s) d s\right\|_{E} \leqslant \frac{1}{c_{0}|z|^{2}}\|f\|_{X} .
$$

Set, for $\varphi \in E$ and $t \in(0,+\infty)$,

$$
B(t, A) \varphi=\frac{1}{2 i \pi} \int_{\gamma} e^{-\sqrt{-z} t}(A-z I)^{-1} \varphi d z .
$$

Then we have the following result, which allows us to study the properties of the solution $u$.

PROPOSITION 2.1. Under Assumption (1.3) we have

(1) that there exists $K>0$ depending only on $\gamma$ such that for all $\varphi \in E$ and for all $t>0,\|B(t, A) \varphi\|_{E} \leqslant K\|\varphi\|_{E}$;

(2) for all $\varphi \in E$ and for all $t>0, B(t, A) \varphi \in D(A)$;

(3) $B(\cdot, A) \varphi \in X$ if and only if $\varphi \in \overline{D(A)}$.

ProOF. (1) For $t>0$, we can write

$$
\begin{aligned}
B(t, A) \varphi & =\frac{1}{2 i \pi} \int_{\gamma_{t}^{+}} e^{-\sqrt{-z} t}(A-z I)^{-1} \varphi d z+\frac{1}{2 i \pi} \int_{\gamma_{t}^{-}} e^{-\sqrt{-z} t}(A-z I)^{-1} \varphi d z \\
& =I_{+}+I_{-},
\end{aligned}
$$


where

$$
\gamma_{t}^{+}=\left\{z \in \gamma:|z| \geqslant 1 / t^{2}\right\}, \quad \gamma_{i}^{-}=\left\{z \in \gamma:|z| \leqslant 1 / t^{2}\right\}
$$

Then

$$
\left\|I_{+}\right\|_{E} \leqslant K \int_{\gamma_{r}^{+}} e^{-(\operatorname{Re} \sqrt{-z}) r} \frac{|d z|}{|z|}\|\varphi\|_{E} \leqslant K \int_{1}^{+\infty} \frac{e^{-c_{0} \sigma}}{\sigma^{2} / t^{2}} \frac{2 \sigma}{t^{2}} d \sigma\|\varphi\|_{E} \leqslant K\|\varphi\|_{E},
$$

and

$$
I_{-}=\frac{1}{2 i \pi} \int_{\gamma_{t}^{-}}\left(e^{-\sqrt{-z} t}-1\right)(A-z I)^{-1} \varphi d z-\frac{1}{2 i \pi} \int_{C_{t}}(A-z I)^{-1} \varphi d z=I_{-}^{\prime}+I_{-}^{\prime \prime},
$$

where $C_{t}=\left\{z=e^{i v} / t^{2}:-\delta_{0} \leqslant v \leqslant \delta_{0}\right\}$. For $I_{-}^{\prime}$, we write

$$
\begin{aligned}
I_{-}^{\prime}= & \frac{1}{2 i \pi} \int_{z \in Y_{t}^{-}, \epsilon_{0} \leqslant|z| \leqslant 1 / t^{2}}\left(e^{-\sqrt{-z} t}-1\right)(A-z I)^{-1} \varphi d z \\
& +\frac{1}{2 i \pi} \int_{z \in Y_{t}^{-}, z=\epsilon_{0} e^{i \nu}}\left(e^{-\sqrt{-z} t}-1\right)(A-z I)^{-1} \varphi d z=J_{-}^{1}+J_{-}^{2},
\end{aligned}
$$

then

$$
\begin{aligned}
& \left\|J_{-}^{1}\right\|_{E} \leqslant K \int_{\epsilon_{0}}^{1 / t^{2}} \frac{\left|e^{-\sqrt{-z} t}-1\right|}{|z|} d|z|\|\varphi\|_{E} \leqslant K \int_{0}^{1 / t^{2}}|z|^{1 / 2} t \frac{d|z|}{|z|}\|\varphi\|_{E} \leqslant K \mid \varphi \|_{E}, \\
& \left\|J_{-}^{2}\right\|_{E} \leqslant \frac{2 \epsilon_{0}}{2 \pi} \int_{\delta_{0}}^{2 \pi-\delta_{0}}\left\|\left(A-\epsilon_{0} e^{i \nu} I\right)^{-1} \varphi\right\|_{E} d v \leqslant K\|\varphi\|_{E} .
\end{aligned}
$$

For $I_{-}^{\prime \prime}$, we have

$$
\left\|I_{-}^{\prime \prime}\right\|_{E} \leqslant K \int_{-\delta_{0}}^{+\delta_{0}}\left\|\left(A-\frac{1}{t^{2}} e^{i v}\right)^{-1} \varphi\right\|_{E} \frac{1}{t^{2}} d v \leqslant K\|\varphi\|_{E} .
$$

(2) This rises from the convergence of the integral

$$
\frac{1}{2 i \pi} \int_{\gamma} e^{-\sqrt{-z} t} A(A-z I)^{-1} \varphi d z
$$

and from the fact that $e^{-\sqrt{-z} t}(A-z I)^{-1} \varphi \in D(A)$ for all $\varphi \in E$ and $t>0$.

(3) Fix $\epsilon>0$ and let $\varphi \in \overline{D(A)}$, then there exists $\psi \in D(A)$ such that

$$
\|\varphi-\psi\|_{E} \leqslant \epsilon
$$


Using the resolvent's identity $(A-z I)^{-1} A \psi=\psi+z(A-z I)^{-1} \psi$, we obtain

$$
B(t, A) \psi=\frac{1}{2 i \pi} \int_{\gamma} \frac{e^{-\sqrt{-z} t}}{z}(A-z I)^{-1} A \psi d z .
$$

Thanks to the inequality

$$
\left\|\frac{e^{-\sqrt{-z} t}}{z}(A-z I)^{-1} A \psi\right\|_{E} \leqslant \frac{K}{|z|^{2}}\|A \psi\|_{E},
$$

Lebesgue's and Cauchy's theorems give us

$$
\lim _{t \rightarrow 0^{+}} B(t, A) \psi=\frac{1}{2 i \pi} \int_{\gamma} \frac{(A-z I)^{-1}}{z} A \psi d z=\psi .
$$

Now from the equality

$$
B(t, A) \varphi-\varphi=(B(t, A) \varphi-B(t, A) \psi)+(B(t, A) \psi-\psi)+(\psi-\varphi),
$$

and from the estimate (2.3), we deduce that $B(t, A) \varphi-\varphi \rightarrow 0$ as $t \rightarrow 0^{+}$. The uniform continuity in $t>0$ is easily verified.

Conversely, if $B(\cdot, A) \varphi \in X$, then for $z \in \gamma$ one has

$$
\begin{aligned}
(A-z I)^{-1} \lim _{t \rightarrow 0^{+}} B(t, A) \varphi & =\lim _{t \rightarrow 0^{+}}(A-z I)^{-1} B(t, A) \varphi \\
& =\lim _{t \rightarrow 0^{+}} B(t, A)(A-z I)^{-1} \varphi \\
& =(A-z I)^{-1} \varphi,
\end{aligned}
$$

wich implies that $\varphi=\lim _{t \rightarrow 0^{+}} B(t, A) \varphi \in \overline{D(A)}$.

PROPOSITION 2.2. Under Assumption (1.3) and for $\theta \in(0,1 / 2)$ we have

$$
B(\cdot, A) \varphi \in C^{2 \theta}(E) \text { if and only if } \varphi \in D_{A}(\theta,+\infty) .
$$

ProOF. Let $\varphi \in D_{A}(\theta,+\infty)$ and $0 \leqslant \tau<t$. Thus

$$
\begin{aligned}
(\tau, A) \varphi-B(t, A) \varphi= & \frac{1}{2 i \pi} \int_{\gamma}\left(e^{-\sqrt{-z} \tau}-e^{-\sqrt{-z} t}\right) \frac{A(A-z I)^{-1}}{z} \varphi d z \\
= & \frac{1}{2 i \pi} \int_{z \in \gamma,|z| \geqslant(t-\tau)^{-2}}\left(e^{-\sqrt{-z} \tau}-e^{-\sqrt{-z} t}\right) \frac{A(A-z I)^{-1}}{z} \varphi d z \\
& +\frac{1}{2 i \pi} \int_{z \in \mathcal{Y},|z| \leqslant(t-\tau)^{-2}}\left(e^{-\sqrt{-z} \tau}-e^{-\sqrt{-z} t}\right) \frac{A(A-z I)^{-1}}{z} \varphi d z,
\end{aligned}
$$


and

$$
\begin{aligned}
&\|B(\tau, A) \varphi-B(t, A) \varphi\|_{E} \\
& \leqslant K \int_{z \in \gamma_{,}|z| \geqslant(t-\tau)^{-2}} \frac{|d z|}{|z|^{\theta+1}}\|\varphi\|_{D_{A}(\theta,+\infty)} \\
&+K^{\prime} \int_{z \in \gamma_{.}|z| \leqslant(t-\tau)^{-2}} \frac{e^{-(\operatorname{Re} \sqrt{-z}) \tau}|z|^{1 / 2}(t-\tau)}{|z|^{\theta+1}}|d z|\|\varphi\|_{D_{A}(\theta,+\infty)} \\
& \leqslant K \int_{z \in \gamma_{.}|z| \geqslant(t-\tau)^{-2}} \frac{|d z|}{|z|^{\theta+1}}\|\varphi\|_{D_{A}(\theta,+\infty)} \\
&+K^{\prime} \int_{z \in \gamma_{,}|z| \leqslant(t-\tau)^{-2}} \frac{|z|^{1 / 2}(t-\tau)}{|z|^{\theta+1}}|d z|\|\varphi\|_{D_{A}(\theta,+\infty)} \\
& \leqslant \max \left(K, K^{\prime}\right)(t-\tau)^{2 \theta}\|\varphi\|_{D_{A}(\theta,+\infty)} .
\end{aligned}
$$

For the proof of the direct sense, we know (see Sinestrari [10]) that if $B$ is the infinitesimal generator of an analytic semigroup $V(t)$, then $V(t) \varphi-\varphi=O\left(t^{\alpha}\right)$, as $t \rightarrow 0^{+}$, if and only if $\varphi \in D_{B}(\alpha,+\infty)$. Observe that $-(-A)^{1 / 2}$ is the infinitesimal generator of the analytic semigroup $V(t)=B(t, A)$ (see Krein [5]). Therefore,

$$
\varphi \in D_{(-A)^{1 / 2}}(2 \theta,+\infty)=D_{A}(\theta,+\infty)
$$

The last equality holds by using the reiteration theorem in interpolation theory (see Lions-Peetre [8]).

\section{Smoothness of the solution}

Now we can state some regularity properties of $u$ and $A u$.

Proposition 3.1. Let $\varphi \in D(A)$ and $f \in C^{2 \theta}(E)$, with $\theta \in(0,1 / 2)$. Then

(1) for all $t \geqslant 0, u(t) \in D(A)$;

(2) $u(\cdot)$ and $u^{\prime}(\cdot) \in X$;

(3) $S(\cdot)=A u(\cdot)-B(\cdot, A)(A \varphi-f(0)) \in C^{2 \theta}(E)$;

(4) $A u(\cdot) \in X$ if and only if $A \varphi-f(0) \in \overline{D(A)}$;

(5) $A u(\cdot) \in C^{2 \theta}(E)$ if and only if $A \varphi-f(0) \in D_{A}(\theta,+\infty)$.

ProOF. (1) In the second integral in (2.1) we write $f(s)=(f(s)-f(t))+f(t)$. Then, after a calculation of the integral in $f(t)$, we get

$$
u(t)=B(t, A) \varphi-\frac{1}{2 i \pi} \int_{\gamma} \int_{0}^{+\infty} k(t, s)(A-z I)^{-1}(f(s)-f(t)) d s d z
$$




$$
\begin{aligned}
& -\frac{1}{2 i \pi} \int_{y} \frac{e^{-\sqrt{-2} t}}{z}(A-z I)^{-1} f(t) d z \\
& +\frac{1}{2 i \pi} \int_{y} \frac{1}{z}(A-z I)^{-1} f(t) d z \\
& =I_{1}+I_{2}+I_{3}+I_{4} .
\end{aligned}
$$

From Proposition 2.1, we deduce that $I_{1} \in D(A)$. The convergence of the integral

$$
\frac{1}{2 i \pi} \int_{\gamma} \frac{e^{-\sqrt{-z} t}}{z} A(A-z I)^{-1} f(t) d z
$$

implies that $I_{3} \in D(A)$. For $I_{4}$ we use the Cauchy theorem, which gives

$$
I_{4}=A^{-1} f(t) \in D(A)
$$

Finally for $I_{2}$, it is sufficient to prove the convergence of the integral

$$
\frac{1}{2 i \pi} \int_{\gamma} \int_{0}^{+\infty} k(t, s) A(A-z I)^{-1}(f(s)-f(t)) d s d z
$$

In fact, we have

$$
\begin{aligned}
& \left\|\frac{1}{2 i \pi} \int_{\gamma} \int_{0}^{+\infty} k(t, s) A(A-z I)^{-1}(f(s)-f(t)) d s d z\right\|_{E} \\
& \quad \leqslant K \int_{\epsilon_{0}}^{+\infty}\left(\sup _{t>0} \int_{0}^{+\infty}|k(t, s) \| t-s|^{2 \theta} d s\right) d|z|\|f\|_{C^{2 \theta}(E)} \\
& \quad \leqslant K \int_{\epsilon_{0}}^{+\infty} \frac{d|z|}{|z|^{1+\theta}}\|f\|_{C^{2 \theta}(E)},
\end{aligned}
$$

where the last estimate holds by Hölder's inequality.

(2) Let us prove that $u(\cdot) \in X$ (the same techniques give the result $u^{\prime}(\cdot) \in X$ ). For $0 \leqslant \tau<t$, we get $u(t)-u(\tau)=l+\Delta_{1}+\Delta_{2}+J$, where

$$
\begin{aligned}
I= & B(t, A) \varphi-B(\tau, A) \varphi, \quad J=A^{-1}(f(t)-f(\tau)), \\
\Delta_{1}= & -\frac{1}{2 i \pi} \int_{\gamma} \int_{0}^{+\infty} k(t, s)(A-z I)^{-1}(f(s)-f(t)) d s d z \\
& +\frac{1}{2 i \pi} \int_{\gamma} \int_{0}^{+\infty} k(\tau, s)(A-z I)^{-1}(f(s)-f(\tau)) d s d z \\
\Delta_{2}= & -\frac{1}{2 i \pi} \int_{\gamma} \frac{e^{-\sqrt{-z} t}}{z}(A-z I)^{-1} f(t) d z+\frac{1}{2 i \pi} \int_{\gamma} \frac{e^{-\sqrt{-z} \tau}}{z}(A-z I)^{-1} f(\tau) d z .
\end{aligned}
$$


The result is obvious for $I$, since $\varphi \in D(A)$. For $J$ we use Assumption (1.3).

On the other hand, we have

$$
\begin{aligned}
\Delta_{2}= & \frac{1}{2 i \pi} \int_{\gamma} \frac{e^{-\sqrt{-z} t}}{z}(A-z I)^{-1}(f(\tau)-f(t)) d z \\
& -\frac{1}{2 i \pi} \int_{\gamma} \frac{\left(e^{-\sqrt{-2} t}-e^{-\sqrt{-z} \tau}\right)}{z}(A-z I)^{-1} f(\tau) d z \\
= & \Delta_{2}^{\prime}+\Delta_{2}^{\prime \prime},
\end{aligned}
$$

where

$$
\left\|\Delta_{2}^{\prime}\right\|_{E} \leqslant\left(\frac{1}{2 \pi} \int_{\epsilon_{0}}^{+\infty} \frac{|d z|}{|z|^{2}}\right)(t-\tau)^{2 \theta}\|f\|_{C^{2 \theta}(E)} \leqslant K(t-\tau)^{2 \theta}\|f\|_{C^{2 \theta}(E)},
$$

and writing $\Delta_{2}^{\prime \prime}$ as

$$
\Delta_{2}^{\prime \prime}=\frac{1}{2 i \pi} \int_{\gamma} \frac{1}{z}\left(\int_{\tau}^{t} \sqrt{-z} e^{-\sqrt{-z} \xi} d \xi\right)(A-z I)^{-1} f(\tau) d z
$$

we obtain

$$
\left\|\Delta_{2}^{\prime \prime}\right\|_{E} \leqslant\left(\frac{1}{2 \pi} \int_{\epsilon_{0}}^{+\infty} \frac{|d z|}{|z|^{3 / 2}}\right)(t-\tau)\|f\|_{X} \leqslant K(t-\tau)\|f\|_{X} .
$$

Now for the quantity $\Delta_{1}$, one has

$$
\begin{aligned}
\Delta_{1}= & -\frac{1}{2 i \pi} \int_{\gamma} \int_{\tau}^{t} \frac{e^{-\sqrt{-z} t} \sinh \sqrt{-z} s}{\sqrt{-z}}(A-z I)^{-1}(f(s)-f(t)) d s d z \\
& +\frac{1}{2 i \pi} \int_{\gamma} \int_{\tau}^{t} \frac{e^{-\sqrt{-z} s} \sinh \sqrt{-z} \tau}{\sqrt{-z}}(A-z I)^{-1}(f(s)-f(\tau)) d s d z \\
& -\frac{1}{2 i \pi} \int_{\gamma} \int_{0}^{\tau} \frac{e^{-\sqrt{-z} t} \sinh \sqrt{-z} s}{\sqrt{-z}}(A-z I)^{-1}(f(\tau)-f(t)) d s d z \\
& -\frac{1}{2 i \pi} \int_{\gamma} \int_{0}^{\tau} \frac{e^{-\sqrt{-z} t}-e^{-\sqrt{-z} \tau}}{\sqrt{-z}} \sinh \sqrt{-z} s(A-z I)^{-1}(f(s)-f(\tau)) d s d z \\
& -\frac{1}{2 i \pi} \int_{\gamma} \int_{t}^{+\infty} \frac{e^{-\sqrt{-z} s} \sinh \sqrt{-z} \tau}{\sqrt{-z}}(A-z I)^{-1}(f(\tau)-f(t)) d s d z \\
& -\frac{1}{2 i \pi} \int_{\gamma} \int_{t}^{+\infty} \frac{(\sinh \sqrt{-z} t-\sinh \sqrt{-z} \tau)}{e^{\sqrt{-z} s} \sqrt{-z}}(A-z I)^{-1}(f(s)-f(t)) d s d z \\
= & \sum_{i=6} I_{i} .
\end{aligned}
$$


Then

$\left\|I_{1}\right\|_{E} \leqslant K \int_{\tau}^{t}\left(\int_{\epsilon_{0}}^{+\infty} \frac{|d z|}{|z|^{3 / 2}}\right)(t-s)^{2 \theta} d s\|f\|_{C^{2 \theta}(E)} \leqslant K(t-\tau)^{2 \theta+1}\|f\|_{C^{2 \theta}(E)}$, and

$\left\|I_{2}\right\|_{E} \leqslant K \int_{\tau}^{t}\left(\int_{\epsilon_{0}}^{+\infty} \frac{|d z|}{|z|^{3 / 2}}\right)(s-\tau)^{2 \theta} d s\|f\|_{C^{2 \theta}(E)} \leqslant K(t-\tau)^{2 \theta+1}\|f\|_{C^{2 \theta}(E)}$.

Writing $I_{4}$ and $I_{6}$ as

$$
\begin{aligned}
& I_{4}=\frac{1}{2 i \pi} \int_{\gamma} \int_{0}^{\tau}\left(\int_{\tau}^{t} e^{-\sqrt{-z} \xi} d \xi\right) \sinh \sqrt{-z} s(A-z I)^{-1}(f(s)-f(\tau)) d s d z, \\
& I_{6}=-\frac{1}{2 i \pi} \int_{\gamma} \int_{t}^{+\infty} e^{-\sqrt{-z} s}\left(\int_{\tau}^{t} \cosh \sqrt{-z} \xi d \xi\right)(A-z I)^{-1}(f(s)-f(t)) d s d z,
\end{aligned}
$$

we get

$$
\begin{aligned}
\left\|I_{4}\right\|_{E} & \leqslant \frac{1}{2 \pi} \int_{\epsilon_{0}}^{+\infty}\left(\int_{0}^{\tau} \int_{\tau}^{t} e^{-\operatorname{Re} \sqrt{-z}(\xi-s)}(\tau-s)^{2 \theta} d \xi d s\right) \frac{|d z|}{1+|z|}\|f\|_{C^{2 \theta}(E)} \\
& \leqslant K \int_{0}^{\tau} \int_{\tau-s}^{t-s} \frac{(\tau-s)^{2 \theta}}{\eta^{2}}\left(\int_{0}^{+\infty} e^{-c_{0} \sigma} 2 \sigma d \sigma\right) d \eta d s\|f\|_{C^{2 \theta}(E)} \\
& \leqslant K \int_{0}^{\tau}(\tau-s)^{2 \theta}\left(\frac{1}{\tau-s}-\frac{1}{t-s}\right) d s\|f\|_{C^{2 \theta}(E)} \\
& \leqslant K(t-\tau) \int_{0}^{\tau} \frac{(\tau-s)^{2 \theta-1}}{(t-\tau+\tau-s)} d s\|f\|_{C^{2 \theta}(E),}
\end{aligned}
$$

by making the change of variable $(\tau-s)=\xi(t-\tau)$, we obtain

$$
\left\|I_{4}\right\|_{E} \leqslant K(t-\tau)^{2 \theta}\left(\int_{0}^{+\infty} \frac{\xi^{2 \theta-1}}{(1+\xi)} d \xi\right)\|f\|_{C^{2 \theta}(E)} \leqslant K(t-\tau)^{2 \theta}\|f\|_{C^{2 \theta}(E)} .
$$

For $I_{6}$, we have

$$
\begin{aligned}
\left\|I_{6}\right\|_{E} & \leqslant K \int_{\epsilon_{0}}^{+\infty} \int_{t}^{+\infty} \int_{\tau}^{t} e^{-\operatorname{Re} \sqrt{-z}(s-\xi)}(s-t)^{2 \theta} d \xi d s \frac{|d z|}{1+|z|}\|f\|_{C^{2 \theta}(E)} \\
& \leqslant K \int_{t}^{+\infty} \int_{\tau}^{t}\left(\int_{0}^{+\infty} e^{-c_{0} \sigma} \sigma d \sigma\right) \frac{(s-t)^{2 \theta}}{(s-\xi)^{2}} d \xi d s\|f\|_{C^{2 \theta}(E)} \\
& \leqslant K \int_{t}^{+\infty}(s-t)^{2 \theta}\left(\int_{s-t}^{s-\tau} \frac{d \eta}{\eta^{2}}\right) d s\|f\|_{C^{2 \theta}(E)} \\
& \leqslant K(t-\tau) \int_{t}^{+\infty} \frac{(s-t)^{2 \theta-1}}{(s-t+t-\tau)} d s\|f\|_{C^{2 \theta}(E)}
\end{aligned}
$$




$$
\begin{aligned}
& \leqslant K(t-\tau)^{2 \theta}\left(\int_{0}^{+\infty} \frac{\rho^{2 \theta-1}}{(1+\rho)} d \rho\right)\|f\|_{C^{2 \theta}(E)} \\
& \leqslant K(t-\tau)^{2 \theta}\|f\|_{C^{2 \theta}(E) .}
\end{aligned}
$$

A direct calculation of the integrals in $(f(\tau)-f(t))$ implies that

$$
\begin{aligned}
I_{3}+I_{5}= & \frac{1}{2 i \pi} \int_{\gamma} \frac{e^{-\sqrt{-z}(t-\tau)}}{z}(A-z I)^{-1}(f(\tau)-f(t)) d z \\
& -\frac{1}{2 i \pi} \int_{\gamma} \frac{e^{-\sqrt{-z} t}}{z}(A-z I)^{-1}(f(\tau)-f(t)) d z \\
= & J-\Delta_{2}^{\prime} .
\end{aligned}
$$

We write $J$ as

$$
\begin{aligned}
J= & \frac{1}{2 i \pi} \int_{\gamma_{(t-\tau)}^{+}} \frac{e^{-\sqrt{-z}(t-\tau)}}{z}(A-z I)^{-1}(f(\tau)-f(t)) d z \\
& +\frac{1}{2 i \pi} \int_{\gamma_{(t-\tau)}^{-}} \frac{e^{-\sqrt{-z}(t-\tau)}}{z}(A-z l)^{-1}(f(\tau)-f(t)) d z \\
= & J^{+}+J^{-}
\end{aligned}
$$

(where $\gamma_{(t-\tau)}^{+}$and $\gamma_{(t-\tau)}^{-}$are defined in (2.2)), then

$$
\begin{aligned}
\left\|J^{+}\right\|_{E} & \leqslant K \int_{1}^{+\infty} \frac{e^{-\operatorname{Re} \sqrt{-z}(t-\tau)}}{|z|} \frac{(t-\tau)^{2 \theta}}{1+|z|}|d z|\|f\|_{C^{2 \theta}(E)} \\
& \leqslant K(t-\tau)^{2 \theta}\left(\int_{1}^{+\infty} \frac{e^{-\operatorname{Re} \sqrt{-z}(t-\tau)}}{|z|}|d z|\right)\|f\|_{C^{2 \theta}(E)} \\
& \leqslant K(t-\tau)^{2 \theta}\left(\int_{1}^{+\infty} \frac{e^{-c_{0} \sigma}}{\sigma} d \sigma\right)\|f\|_{C^{2 \theta}(E)} \\
& \leqslant K(t-\tau)^{2 \theta}\|f\|_{C^{2 \theta}(E)}
\end{aligned}
$$

and for $J^{-}$we have

$$
\begin{aligned}
J^{-}= & \frac{1}{2 i \pi} \int_{\gamma_{(t-\tau)}^{-}}\left(e^{-\sqrt{-z}(t-\tau)}-1\right) \frac{(A-z I)^{-1}(f(\tau)-f(t))}{z} d z \\
& +A^{-1}(f(\tau)-f(t))-\frac{1}{2 i \pi} \int_{C_{(t-\tau)}} \frac{(A-z I)^{-1}(f(\tau)-f(t))}{z} d z \\
= & J_{1}^{-}+A^{-1}(f(\tau)-f(t))+J_{2}^{-},
\end{aligned}
$$

with $C_{(t-\tau)}=\left\{z=(t-\tau)^{-2} e^{i v}:-\delta_{0} \leqslant v \leqslant \delta_{0}\right\}$. 
So we obtain

$$
\begin{aligned}
\left\|J_{1}^{-}\right\|_{E} & \leqslant K \int_{0}^{1 /(t-\tau)^{2}} \frac{|z|^{1 / 2}(t-\tau)}{|z|} \frac{(t-\tau)^{2 \theta}}{1+|z|}|d z|\|f\|_{C^{2 \theta}(E)} \\
& \leqslant K(t-\tau)^{2 \theta+1} \int_{0}^{1 /(t-\tau)^{2}} \frac{|z|^{1 / 2}}{|z|}|d z|\|f\|_{C^{2 \theta}(E)} \leqslant K(t-\tau)^{2 \theta}\|f\|_{C^{2 \theta}(E)},
\end{aligned}
$$

and

$\left\|J_{2}^{-}\right\|_{E} \leqslant K \frac{(t-\tau)^{2 \theta}}{1+(t-\tau)^{-2}} \int_{-\delta_{0}}^{\delta_{0}}(t-\tau)^{2} \frac{d v\|f\|_{C^{2 \theta}(E)}}{(t-\tau)^{2}} \leqslant K(t-\tau)^{2 \theta}\|f\|_{C^{2 \theta}(E)}$.

(3) We have

$$
\begin{aligned}
S(t)= & \frac{1}{2 i \pi} \int_{\gamma} \frac{e^{-\sqrt{-z} t}}{z} A(A-z I)^{-1} A \varphi d z \\
& -\frac{1}{2 i \pi} \int_{\gamma} \int_{0}^{+\infty} k(t, s) A(A-z I)^{-1}(f(s)-f(t)) d s d z \\
& -\frac{1}{2 i \pi} \int_{\gamma} \frac{e^{-\sqrt{-z} t}}{z} A(A-z I)^{-1} f(t) d z+f(t) \\
& -\frac{1}{2 i \pi} \int_{\gamma} \frac{e^{-\sqrt{-z} t}}{z} A(A-z I)^{-1}(A \varphi-f(0)) d z,
\end{aligned}
$$

thus

$$
\begin{aligned}
S(t)= & \frac{1}{2 i \pi} \int_{\gamma} \frac{e^{-\sqrt{-z} t}}{z} A(A-z I)^{-1} f(0) d z \\
& -\frac{1}{2 i \pi} \int_{\gamma} \int_{0}^{+\infty} k(t, s) A(A-z I)^{-1}(f(s)-f(t)) d s d z \\
& -\frac{1}{2 i \pi} \int_{\gamma} \frac{e^{-\sqrt{-z} t}}{z} A(A-z I)^{-1} f(t) d z+f(t) .
\end{aligned}
$$

Let $0 \leqslant \tau<t$, then $S(t)-S(\tau)=f(t)-f(\tau)+\Lambda+\Pi$, where

$$
\begin{aligned}
\Lambda= & \frac{1}{2 i \pi} \int_{\gamma} \int_{0}^{+\infty} k(\tau, s) A(A-z I)^{-1}(f(s)-f(\tau)) d s d z \\
& -\frac{1}{2 i \pi} \int_{\gamma} \int_{0}^{+\infty} k(t, s) A(A-z I)^{-1}(f(s)-f(\bar{t})) d s d z,
\end{aligned}
$$

and

$$
\Pi=\frac{1}{2 i \pi}\left(\int_{\gamma} \frac{e^{-\sqrt{-z} t}}{z} A(A-z I)^{-1} f(0) d z-\int_{\gamma} \frac{e^{-\sqrt{-z} t}}{z} A(A-z I)^{-1} f(t) d z\right)
$$


[13]

$$
-\frac{1}{2 i \pi}\left(\int_{\gamma} \frac{e^{-\sqrt{-z} \tau}}{z} A(A-z I)^{-1} f(0) d z-\int_{\gamma} \frac{e^{-\sqrt{-z} \tau}}{z} A(A-z I)^{-1} f(\tau) d z\right) .
$$

Regarding the quantity $\Pi$, we have

$$
\begin{aligned}
\Pi= & \frac{1}{2 i \pi} \int_{\gamma} \frac{\left(e^{-\sqrt{-z} t}-e^{-\sqrt{-z} \tau}\right)}{z} A(A-z I)^{-1} f(0) d z \\
& +\frac{1}{2 i \pi} \int_{\gamma} \frac{e^{-\sqrt{-z} \tau}}{z} A(A-z I)^{-1} f(\tau) d z \\
& +\frac{1}{2 i \pi} \int_{\gamma} \frac{e^{-\sqrt{-z} t}}{z} A(A-z I)^{-1} f(\tau) d z \\
& -\frac{1}{2 i \pi} \int_{\gamma} \frac{e^{-\sqrt{-z} t}}{z} A(A-z I)^{-1} f(\tau) d z \\
& -\frac{1}{2 i \pi} \int_{\gamma} \frac{e^{-\sqrt{-z} t}}{z} A(A-z I)^{-1} f(t) d z \\
= & -\frac{1}{2 i \pi} \int_{\gamma} \frac{\left(e^{-\sqrt{-z} t}-e^{-\sqrt{-z} \tau}\right)}{z} A(A-z I)^{-1}(f(\tau)-f(0)) d z \\
& +\frac{1}{2 i \pi} \int_{\gamma} \frac{e^{-\sqrt{-z} t}}{z} A(A-z I)^{-1}(f(\tau)-f(t)) d z \\
= & \Pi_{1}+\Pi_{2} .
\end{aligned}
$$

From Proposition 2.1, we deduce that

$$
\left\|\Pi_{2}\right\|_{E}=\|B(t, A)(f(\tau)-f(t))\|_{E} \leqslant K(t-\tau)^{2 \theta}\|f\|_{C^{2 \theta}(E)} .
$$

For $\Pi_{1}$, we get

$$
\left\|\Pi_{1}\right\|_{E} \leqslant K \int_{\tau}^{t} \int_{\epsilon_{0}}^{+\infty} \frac{|z|^{1 / 2} e^{-c_{0}|z|^{1 / 2} s} \tau^{2 \theta}}{|z|}|d z| d s\|f\|_{C^{2 \theta}(E)},
$$

and setting $|z|^{1 / 2} s=\sigma$ in this last inequality, we obtain

$$
\begin{aligned}
\left\|\Pi_{1}\right\|_{E} & \leqslant K \int_{\tau}^{t} \frac{\tau^{2 \theta}}{s}\left(\int_{0}^{+\infty} e^{-c_{0} \sigma} d \sigma\right) d s\|f\|_{C^{2 \theta}(E)} \leqslant K\left(\int_{\tau}^{t} s^{2 \theta-1} d s\right)\|f\|_{C^{2 \theta}(E)} \\
& \leqslant K\left(t^{2 \theta}-\tau^{2 \theta}\right)\|f\|_{C^{2 \theta}(E)} \leqslant K(t-\tau)^{2 \theta}\|f\|_{C^{2 \theta}(E) .}
\end{aligned}
$$

$\Lambda$ may be written as

$$
\Lambda=-\frac{1}{2 i \pi} \int_{\gamma} \int_{\tau}^{t} \frac{e^{-\sqrt{-z} t} \sinh \sqrt{-z} s}{\sqrt{-z}} A(A-z I)^{-1}(f(s)-f(t)) d s d z
$$




$$
\begin{aligned}
& +\frac{1}{2 i \pi} \int_{\gamma} \int_{\tau}^{t} \frac{e^{-\sqrt{-z} s} \sinh \sqrt{-z} \tau}{\sqrt{-z}} A(A-z I)^{-1}(f(s)-f(\tau)) d s d z \\
& -\frac{1}{2 i \pi} \int_{\gamma} \int_{0}^{\tau} \frac{e^{-\sqrt{-z} t} \sinh \sqrt{-z} s}{\sqrt{-z}} A(A-z I)^{-1}(f(\tau)-f(t)) d s d z \\
& -\frac{1}{2 i \pi} \int_{\gamma} \int_{0}^{\tau} \frac{\left(e^{-\sqrt{-z} t}-e^{-\sqrt{-z} \tau}\right)}{\sqrt{-z}} \sinh \sqrt{-z} s \\
& \quad \times A(A-z I)^{-1}(f(s)-f(\tau)) d s d z \\
& -\frac{1}{2 i \pi} \int_{\gamma} \int_{t}^{+\infty} \frac{e^{-\sqrt{-z} s} \sinh \sqrt{-z} \tau}{\sqrt{-z}} A(A-z I)^{-1}(f(\tau)-f(t)) d s d z \\
& \quad \frac{1}{2 i \pi} \int_{\gamma} \int_{t}^{+\infty} \frac{e^{-\sqrt{-z} s}(\sinh \sqrt{-z} t-\sinh \sqrt{-z} \tau)}{\sqrt{-z}} \\
& =\sum_{i=1} A(A-z I)^{-1}(f(s)-f(t)) d s d z
\end{aligned}
$$

For $\Lambda_{1}$ and $\Lambda_{2}$, we prove that

$$
\begin{aligned}
\left\|\Lambda_{1}\right\|_{E} & \leqslant K \int_{\epsilon_{0}}^{+\infty} \int_{\tau}^{t} \frac{e^{-\operatorname{Re} \sqrt{-z}(t-s)}}{|z|^{1 / 2}}|t-s|^{2 \theta} d s|d z|\|f\|_{C^{2 \theta}(E)} \\
& \leqslant K \int_{\tau}^{t}(t-s)^{2 \theta-1}\left(\int_{0}^{+\infty} e^{-c_{0} \sigma} d \sigma\right) d s\|f\|_{C^{2 \theta}(E)} \\
& \leqslant K(t-\tau)^{2 \theta}\|f\|_{C^{2 \theta}(E)}, \\
\left\|\Lambda_{2}\right\|_{E} & \leqslant K \int_{\epsilon_{0}}^{+\infty} \int_{\tau}^{t} \frac{e^{-\operatorname{Re} \sqrt{-z}(s-\tau)}}{|z|^{1 / 2}}|s-\tau|^{2 \theta} d s|d z|\|f\|_{C^{2 \theta}(E)} \\
& \leqslant K \int_{\tau}^{t}(s-\tau)^{2 \theta-1}\left(\int_{0}^{+\infty} e^{-c_{0} \sigma} d \sigma\right) d s\|f\|_{C^{2 \theta}(E)} \\
& \leqslant K(t-\tau)^{2 \theta}\|f\|_{C^{2 \theta}(E)} .
\end{aligned}
$$

Writing $\Lambda_{4}$ and $\Lambda_{6}$ as

$$
\begin{aligned}
& \Lambda_{4}=\frac{1}{2 i \pi} \int_{\gamma} \int_{0}^{\tau}\left(\int_{\tau}^{t} e^{-\sqrt{-z} \xi} d \xi\right) \sinh \sqrt{-z} s A(A-z I)^{-1}(f(s)-f(\tau)) d s d z, \\
& \Lambda_{6}=-\frac{1}{2 i \pi} \int_{\gamma} \int_{t}^{+\infty} e^{-\sqrt{-z} s}\left(\int_{\tau}^{t} \cosh \sqrt{-z} \xi d \xi\right) A(A-z I)^{-1}(f(s)-f(t)) d s d z,
\end{aligned}
$$

we obtain

$$
\left\|\Lambda_{4}\right\|_{E} \leqslant K \int_{\epsilon_{0}}^{+\infty} \int_{0}^{\tau} \int_{\tau}^{t} e^{-\operatorname{Re} \sqrt{-z}(\xi-s)}(\tau-s)^{2 \theta} d \xi d s|d z|\|f\|_{C^{2 \theta}(E)}
$$




$$
\begin{aligned}
& \leqslant K \int_{0}^{\tau} \int_{\tau-s}^{t-s} \frac{(\tau-s)^{2 \theta}}{\eta^{2}}\left(\int_{0}^{+\infty} e^{-c_{0} \sigma} 2 \sigma d \sigma\right) d \eta d s\|f\|_{C^{2 \theta}(E)} \\
& \leqslant K(t-\tau)^{2 \theta}\|f\|_{C^{2 \theta}(E)},
\end{aligned}
$$

and

$$
\begin{aligned}
\left\|\Lambda_{6}\right\|_{E} & \leqslant \frac{1}{2 \pi} \int_{\epsilon_{0}}^{+\infty} \int_{t}^{+\infty} \int_{\tau}^{t} e^{-\operatorname{Re} \sqrt{-z}(s-\xi)}(s-t)^{2 \theta} d \xi d s|d z|\|f\|_{C^{2 \theta}(E)} \\
& \leqslant K \int_{t}^{+\infty} \int_{\tau}^{t}\left(\int_{0}^{+\infty} e^{-c_{0} \sigma} \sigma d \sigma\right) \frac{(s-t)^{2 \theta}}{(s-\xi)^{2}} d \xi d s\|f\|_{C^{2 \theta}(E)} \\
& \leqslant K \int_{t}^{+\infty}(s-t)^{2 \theta}\left(\int_{s-t}^{s-\tau} \frac{d \eta}{\eta^{2}}\right) d s\|f\|_{C^{2 \theta}(E)} \\
& \leqslant K(t-\tau)^{2 \theta}\|f\|_{C^{2 \theta}(E) .}
\end{aligned}
$$

By calculating the integrals in $(f(\tau)-f(t))$, we get

$$
\begin{aligned}
\Lambda_{3}+\Lambda_{5}= & \frac{1}{2 i \pi} \int_{\gamma} \frac{e^{-\sqrt{-z}(t-\tau)}}{z} A(A-z I)^{-1}(f(\tau)-f(t)) d z \\
& -B(t, A)(f(\tau)-f(t)) \\
= & Q-\Pi_{2},
\end{aligned}
$$

where $Q=B(t-\tau, A)(f(\tau)-f(t))$. Proposition 2.1 implies that

$$
\|Q\|_{E} \leqslant K(t-\tau)^{2 \theta}\|f\|_{C^{2 \theta}(E)} .
$$

(4) Assume that $A \varphi-f(0) \in \overline{D(A)}$. Then, by Proposition 2.1

$$
A u(\cdot)=S(\cdot)+B(\cdot, A)(A \varphi-f(0)) \in X .
$$

Conversely, if $A u(\cdot) \in X$, the function $B(\cdot, A)(A \varphi-f(0))=A u(\cdot)-S(\cdot)$, is in $X$. Now using Proposition 2.1, we obtain $A \varphi-f(0) \in \overline{D(A)}$.

(5) Let us suppose that $A \varphi-f(0) \in D_{A}(\theta,+\infty)$. By Proposition 2.1, $A u(\cdot) \in$ $C^{2 \theta}(E)$. Conversely, if $A u(\cdot) \in C^{2 \theta}(E)$, then $B(\cdot, A)(A \varphi-f(0)) \in C^{2 \theta}(E)$, and $A \varphi-f(0) \in D_{A}(\theta,+\infty)$.

REMARK. By using the same methods and techniques of calculation, we have a similar result to Proposition 3.1, when the right-hand term of the equation has a spatial smoothness, that is, for all $t \geqslant 0, f(t) \in D_{A}(\theta,+\infty)$, $\sup _{t \geqslant 0}\|f(t)\|_{D_{A}(\theta,+\infty)}<\infty$, with $\theta \in(0,1 / 2)$. See [2] for details.

Now, we can deduce our main results concerning the regularity of $u$. 
THEOREM 3.2. Let $\varphi \in D(A)$ and $f \in C^{2 \theta}(E)$, with $\theta \in(0,1 / 2)$ such that

$$
f(0)-A \varphi \in \overline{D(A)} \text {. }
$$

Then $u$, given in (2.1), is a strict solution of (1.1)-(1.2).

By the continuity of $B(\cdot, A) \varphi$ and Lebesgue's theorem we can verify that $u(0)=\varphi$ and $u(+\infty)=0$. On the other hand, we prove that $u$ verifies (1.1) by using Dunford's operational calculus.

Finally, by Proposition 3.1, the solution $u$ belongs to

$$
B U C^{2}([0,+\infty[; E) \cap B U C([0,+\infty[; D(A)) .
$$

Furthermore, if $f(0)-A \varphi \in D_{A}(\theta,+\infty)$, then we have more regularity on $A u(\cdot)$ and $u^{\prime \prime}(\cdot)$.

THEOREM 3.3. Let $\varphi \in D(A)$ and $f \in C^{2 \theta}(E)$, with $\theta \in(0,1 / 2)$ such that

$$
f(0)-A \varphi \in D_{A}(\theta,+\infty) .
$$

Then the unique strict solution of (1.1)-(1.2) satisfies the property of maximal regularity $A u(\cdot), u^{\prime \prime}(\cdot) \in C^{2 \theta}(E)$.

PROOF. It suffices to apply the previous results, using the fact that $D_{A}(\theta,+\infty) \subset$ $\overline{D(A)}$.

\section{Example}

We give an example governed by (1.1)-(1.2). Consider $E=C([0,1])$ and

$$
\begin{aligned}
D(A) & =\left\{v \in C^{2}([0,1]): v(0)=v(1)=0\right\}, \\
A v & =v^{\prime \prime} .
\end{aligned}
$$

It is easy to check that $A$ satisfies Assumption (1.3). We can thus apply our results to Laplacian problem in an infinite interval, given by

$$
\begin{cases}\frac{\partial^{2} u}{\partial t^{2}}(t, x)+\frac{\partial^{2} u}{\partial x^{2}}(t, x)=f(t, x), & (t, x) \in(0,+\infty) \times(0,1), \\ u(0, x)=\varphi(x), & x \in(0,1), \\ u(+\infty, x)=0, & x \in(0,1), \\ u(t, 0)=u(t, 1)=0 . & \end{cases}
$$


Observe that conditions $\varphi \in D(A)$ and $f(0)-A \varphi \in \overline{D(A)}$, become

$$
\begin{aligned}
& \varphi \in C^{2}([0,1]): \varphi(0)=\varphi(1)=0, \\
& f(0, \cdot)-\varphi^{\prime \prime}(\cdot) \in C([0,1]) \quad \text { and } \\
& f(0,0)-\varphi^{\prime \prime}(0)=f(0,1)-\varphi^{\prime \prime}(1)=0 .
\end{aligned}
$$

The interpolation space $D_{A}(\theta,+\infty)$ is given by

$$
D_{A}(\theta,+\infty)= \begin{cases}\left\{v \in C^{2 \theta}([0,1]): v(0)=v(1)=0\right\} & \text { if } 2 \theta<1, \\ C^{1, *}([0,1]), & \text { if } 2 \theta=1, \\ \left\{v \in C^{1,2 \theta-1}([0,1]): v(0)=v(1)=0\right\} & \text { if } 2 \theta>1,\end{cases}
$$

where $C^{1, *}([0,1])$ is a Zigmund space (see, for example, [12]). Applying Theorem 3.2 and Theorem 3.3 we have the following results.

THEOREM 4.1. Let $f \in C^{2 \theta}([0,+\infty[; C([0,1]))$, with $2 \theta \in(0,1)$, be such that the conditions (4.2) are satisfied. Then Problem (4.1) has a unique solution $u$ satisfying $u \in B U C^{2}\left(\left[0,+\infty[; C([0,1])) \cap B U C\left(\left[0,+\infty\left[; C^{2}([0,1])\right)\right.\right.\right.\right.$.

THEOREM 4.2. Let $f \in C^{2 \theta}([0,+\infty[; C([0,1])$, with $2 \theta \in(0,1)$, be such that

$$
\left\{\begin{array}{l}
\varphi \in C^{2}([0,1]): \varphi(0)=\varphi(1)=0 \\
f(0, \cdot)-\varphi^{\prime \prime}(\cdot) \in C^{2 \theta}([0,1]) \quad \text { and } \\
f(0,0)-\varphi^{\prime \prime}(0)=f(0,1)-\varphi^{\prime \prime}(1)=0
\end{array}\right.
$$

Then Problem (4.1) has a unique solution

$$
u \in B U C^{2}\left(\left[0,+\infty[; C([0,1])) \cap B U C\left(\left[0,+\infty\left[; C^{2}([0,1])\right)\right.\right.\right.\right.
$$

Moreover, $u$ satisfies the maximal regularity

$$
\partial_{x}^{2} u(\cdot, x), \partial_{t}^{2} u(\cdot, x) \in C^{2 \theta}([0,+\infty[; C([0,1]))
$$

\section{References}

[1] A. V. Balakrishnan, 'Fractional powers of closed operators and the semi-groups generated by them', Pacif. J. Math. 10 (1960), 419-437.

[2] T. Berroug, Sur des Problèmes Elliptiques et Paraboliques dans les Espaces de Hölder et les Petits Hölder (Thèse de Doctorat, Université du Havre, France, 2003). 
[3] A. Favini, R. Labbas, S. Maingot, H. Tanabe and A. Yagi, 'Complete abstract differential equations of elliptic type in UMD spaces', Funkcialaj Ekvacioj, 49 (2006), 193-214.

[4] P. Grisvard, 'Spazi di tracce e applicazioni', Rend. Mat. 5 (1972), 657-729.

[5] S. G. Krein, Linear differential equations in Banach space, Translations of Mathematical Monographs 29 (Amer. Math. Soc., Providence, RI, 1971).

[6] A. V. Kuyazyuk, 'The Dirichlet problem for second order differential equations with operator coefficient', Ukraĭn. Mat. Zh. 37 (1985), 256-273 (Russian).

[7] R. Labbas, Problèmes aux Limites pour une Equation Différentielle Abstraite du Second Ordre (Thèse d'état, Université de Nice, France, 1987).

[8] J. L. Lions and J. Peetre, 'Sur une classe d'espaces d'interpolation', Inst. Hautes Etudes Sci. Publ. Math. 19 (1964), 5-86.

[9] G. Da Prato and P. Grisvard, 'Sommes d'opérateurs linéaires et equations différentielles opérationnelles', J. Math. Pures Appl. 54 (1975), 305-387.

[10] E. Sinestrari, 'On the abstract Cauchy problem of parabolic type in spaces of continuous functions', J. Math. Anal. Appl. 66 (1985), 16-66.

[11] P. E. Sobolevskii, 'On equations of parabolic type in Banach space', Trudy Mosc. Mat. Obsch. 10 (1961), 297-350, (in Russian). English transl.: Amer. Math. Soc. Transl. (1965), 1-62.

[12] H. Triebel, Interpolation theory, function spaces, differential operators (North Holland, Amsterdam, 1978).

[13] L. Veron, 'Equations d'evolution semi-linéaires du second ordre dans $L^{1}$, Rev. Roumaine Math. Pures Appl. 27 (1982), 95-123.

Université du Havre

LMAH, BP 540

25 rue Philippe Lebon

76058 Le Havre cedex

France

e-mail: berroug.tarik@voila.fr rabah.labbas@univ-lehavre.fr
Lab. E.D.P. and Hist. of Maths Dept of Mathematics Ecole Normale Supérieure 16050-Kouba, Algiers Algeria

e-mail: sadallah@ens-kouba.dz 